New Theor $\mathrm{J}$

ISSN: 2149-1402
37 (2021) 1-7

Journal of New Theory

https://dergipark.org.tr/en/pub/jnt

Open Access

\title{
Some Results of Common Fixed Point for Compatible Mappings in F- Metric Spaces
}

\author{
Demet Binbaşığlu1
}

\begin{abstract}
Article History
Received: 14 Oct 2021

Abstract - Recently, F-metric space has been started, and a natural topology has been described in these spaces by Jleli and Samet. Furthermore, a new form of the Banach contraction principle has been

Accepted: 29 Dec 2021 given in the new spaces. In this work, we present some common fixed-point theorems for two weakly

Published: 31 Dec 2021 compatible mappings in the F-metric spaces. We also mention examples that confirm our results.
\end{abstract}

10.53570/jnt.1009413

Research Article

Keywords - Common fixed point, F-metric space, weakly compatible

Mathematics Subject Classification (2020) - 47H10, 54A20

\section{Introduction}

Metric space is the general theory underlying various branches of mathematics. These days, generalizations of the metric space have emerged. Lately, some authors have given various generalizations of metric spaces. This situation allows authors to find new work areas. Czerwik described the concept of $b$-metric [1]. Khamsi and Hussain redefined the $b$-metric concept, and they called it metric-type [2]. Fagin et al. gave s-relaxed metric

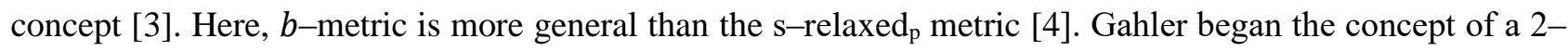
metric [5]. This metric function is identified on the product set $X \times X \times X$. The notion of 2-metric is a generalization of the usual metric. Mustafa and Sims defined the $\mathcal{G}$-metric space concept [6]. The notion is more general than the usual metric. Branciari proposed a new extension of the notion of metric, modified the triangle inequality (iii) with a more general inequality confusing four points. Matthews defined the partial metric [7]. Jleli and Samet defined the JS-metric [8]. Currently, Jleli and Samet have given the F-metric space [9]. They compare their concepts with existing generalizations in the literature. Then, they define a natural topology $\tau_{F}$ on these spaces and examine their topological properties. Also, a new version of the Banach contraction principle is created in the tuning of F-metric spaces. They proved that their new concept is more general than the standard metric concept by showing that any metric space is an F-metric space, but the reverse is generally false. They also compared their concept with previous generalizations of metric spaces. After that, Alnaser et al. gave relation theoretic contraction and proved some generalization of fixed-point theorems in these spaces [10]. Moreover, in these generalized spaces, the coincidence points and common fixed-point

\footnotetext{
${ }^{1}$ demet.binbasioglu@gop.edu.tr (Corresponding Author)

${ }^{1}$ Department of Mathematics, Faculty of Arts and Sciences, Tokat Gaziosmanpaşa University, Tokat, Turkey
} 
theorems are also frequently studied [11]. In this paper, we give some common fixed-point theorems and results in F-metric spaces. Moreover, some examples that provide the theorems are presented.

\section{Preliminaries}

Definition 2.1. [9] Let $F$-be the set of function $g:(0, \infty) \rightarrow \mathbb{R}$. This function provides the below terms.

$\mathrm{F}_{1} . g$ be a non-decreasing function

F2. $\lim _{n \rightarrow \infty} a_{n}=0 \Leftrightarrow \lim _{n \rightarrow \infty} g\left(a_{n}\right)=-\infty$, for every sequence $\left\{a_{n}\right\} \subseteq(0+\infty)$.

Definition 2.2. [9] Let $X \neq \emptyset, D: X \times X \rightarrow[0, \infty)$ is a mapping, and there exist $g \in \mathrm{F}$ and $\gamma \in[0, \infty)$. If the following terms are satisfied, $D$ be defined as an F-metric on $X$. In this case, $(X, D)$ is defined as an F-metric space.

$D_{1 .}(a, b) \in X \times X, a=b \Leftrightarrow D(a, b)=0$,

$D_{2 .} D(a, b)=D(b, a)$ for all $a, b \in X$,

$D_{3 .} \forall a, b \in X, n \in \mathbb{N}, n \geq 2$ and $\left(t_{i}\right)_{i=1}^{n} \subset X$ with $\left(t_{1}, t_{n_{0}}\right)=(a, b)$ we have

$$
D(a, b)>0 \Rightarrow g(D(a, b)) \leq g\left(\sum_{i=1}^{n-1} D\left(t_{i}, t_{i+1}\right)\right)+\gamma
$$

Remark 2.3. [9] The metric space is an F-metric space. But the contrary of this proposition is false.

Example 2.4. [9] Let $\mathbb{N}$ be positive real numbers set. $D: \mathbb{N} \times \mathbb{N} \rightarrow[0, \infty)$ be the mapping and for all $a, b \in \mathbb{N}$,

$$
D(a, b)=\left\{\begin{array}{cc}
(a-b)^{2}, & a, b \in[0,3] \\
|a-b|, & a, b \notin[0,3]
\end{array}\right.
$$

Therefore, $D$ is an F-metric with $g(a)=\ln a, a>0$ and $\gamma=\ln 3$.

Example 2.5. [9] Let $\mathbb{N}$ be the natural numbers set, $D: \mathbb{N} \times \mathbb{N} \rightarrow[0, \infty)$ and for all $a, b \in \mathbb{N}$,

$$
D(a, b)=\left\{\begin{array}{cc}
\exp (|a-b|), & a \neq b \\
0, & a=b
\end{array}\right.
$$

Therefore, $D$ is an F-metric with $g(a)=-1 / a, a>0$ and $\gamma=1$.

Definition 2.6. [9] Suppose that $D$ be an F-metric on $X,\left\{a_{n}\right\} \subset X$ is a sequence.

$i$. If $\left\{a_{n}\right\}$ be convergent to an element according to F-metric $D,\left\{a_{n}\right\}$ is $\mathrm{F}$-convergent to element $a$.

ii. If $\lim _{m, n \rightarrow \infty} D\left(a_{n}, a_{m}\right)=0$ then the sequence $\left\{a_{n}\right\}$ is F-Cauchy.

iii. If any $\mathrm{F}$-Cauchy sequence be convergent, $(X, D)$ is $\mathrm{F}$-complete.

Definition 2.7. [11] Let $T$ and $S$ be self-maps on a set $X$. If $T x=S x=y$ for some $x \in X$, then $x, y$ are defined as a coincident point and a coincidence point, respectively. If $x=T x=S x$ for some $x \in X, x$ defined as a common fixed point.

Remark 2.8. [11] If $T$ and $S$ are weakly compatible, then the coincidence point $y$ is the unique common fixed point.

Theorem 2.9. [9] Let $(X, D)$ be an F-metric space, $f: X \rightarrow X$. Assume that the F-metric space $(X, D)$ is $\mathrm{F}$ complete and there exists $\alpha \in(0,1)$ such that $D(f(a), f(b)) \leq \alpha D(a, b)$ for $a, b \in X$. Then $f$ has a unique fixed point $a^{*} \in X$. Furthermore, the sequence $\left\{a_{n}\right\} \subset X$ given by $a_{n+1}=f\left(a_{n}\right), n \in \mathbb{N}$ is F-convergent to $a^{*}$, for any $a_{0} \in X$. 
Remark 2.10. In the theorems and results we have given throughout the article; we will think that the map $g \in \mathrm{F}$ is surjective and $\gamma=0$ for the proof to proceed smoothly.

\section{Main Results}

In this part, we give generalizations of some known fixed-point theorems in the F-metric spaces. These are coincidence points and common fixed-point theorems. Moreover, we denote some examples of the presented results.

Theorem 3.1. Let $(X, D)$ be an F-metric. Assume that $S, \mathrm{~T}: X \rightarrow X$ provides the below conditions

$i$. For $\forall a, b \in X$,

$$
\begin{aligned}
D(T(a), T(b)) \leq & k D(S(a), T(a))+l D(S(b), T(b))+m D(S(a), T(b))+p D(S(b), T(a)) \\
& +t D(S(a), S(b))
\end{aligned}
$$

where $k, l, m, p$, and $t$ are non-negative and $k+l+m+p+t<1$,

ii. $T(X) \subset S(X)$,

iii. $T(X)$ or $S(X)$ be an F-complete subspace.

Therefore, $T$ and $S$ have a unique coincidence point.

Furthermore, if $T$ and $S$ are weakly compatible, they have a unique common fixed point.

Proof.

Let $g \in \mathrm{F}, \gamma=0$ be such that for every $a, b \in X$ for $\forall n \in \mathbb{N}, n \geq 2$ and for $\forall\left(t_{i}\right)_{i=1}^{n} \subset X$ with $\left(t_{1}, t_{n_{0}}\right)=$ $(a, b)$, we have

$$
D(a, b)>0 \Rightarrow g(D(a, b)) \leq g\left(\sum_{i=1}^{n-1} D\left(\left(t_{i}, t_{i+1}\right)\right)+\gamma\right.
$$

From $\mathrm{F}_{2}$, for every sequence $\left\{a_{n}\right\} \subseteq(0,+\infty)$, there exists a $\varepsilon>0$ such that

$$
\lim _{n \rightarrow \infty} a_{n}=0 \Leftrightarrow \lim _{n \rightarrow \infty} g\left(a_{n}\right)=-\infty \text { and } 0<a<\varepsilon \Rightarrow g(a)<g(\varepsilon)-\gamma
$$

Let $a_{0}, a_{1} \in X$ be arbitrary and $\left\{a_{n}\right\} \subset X$ be the sequence defined by $S a_{n+1}=T a_{n}=b_{n}$ for $n \in \mathbb{N}$. We have that

and

$$
D\left(b_{n}, b_{n+1}\right) \leq(k+t) D\left(b_{n-1}, b_{n}\right)+l D\left(b_{n}, b_{n+1}\right)+m D\left(b_{n-1}, b_{n+1}\right)
$$

$$
D\left(b_{n+1}, b_{n}\right) \leq k D\left(b_{n}, b_{n+1}\right)+(l+m) D\left(b_{n-1}, b_{n}\right)+p D\left(b_{n-1}, b_{n+1}\right)
$$

for all $n$. Hence, from the above remark,

$$
D\left(b_{n}, b_{n+1}\right) \leq \frac{k+l+t+p+2 m}{2-(k+l+m+p)} D\left(b_{n-1}, b_{n}\right)
$$

If we choose $c=\frac{k+l+t+p+2 m}{2-(k+l+m+p)}$, then $c \in[0,1)$ and $D\left(b_{n}, b_{n+1}\right) \leq c D\left(b_{n-1}, b_{n}\right)$ is hold. We have

Thus, for all $n$ and $z$,

$$
D\left(b_{n}, b_{n+1}\right) \leq c^{n} D\left(b_{0}, b_{1}\right)
$$

$$
\begin{aligned}
D\left(b_{n}, b_{n+z}\right) & \leq D\left(b_{n}, b_{n+1}\right)+D\left(b_{n+1}, b_{n+2}\right)+\cdots+D\left(b_{n+z-1}, b_{n+z}\right) \\
& \leq\left(c^{n}+c^{n+1}+\cdots+c^{n+z-1}\right) D\left(b_{0}, b_{1}\right) \\
& \leq \frac{c^{n}}{1-c} D\left(b_{0}, b_{1}\right)
\end{aligned}
$$

holds. 
Since $\lim _{n \rightarrow \infty} \frac{c^{n}}{1-c} D\left(b_{0}, b_{1}\right)=0$, there exists a $n_{0} \in \mathbb{N}$ such that $0<\frac{c^{n}}{1-c} D\left(b_{0}, b_{1}\right)<\varepsilon, n \geq n_{0}$. From conditions $0<b<\varepsilon \Rightarrow g(b)<g(\varepsilon)-\gamma$ and since $g$ is non-decreasing.

$$
g\left(\sum_{i=n}^{n+z-1} D\left(b_{i}, b_{i+1}\right)\right) \leq g\left(\frac{c^{n}}{1-c} D\left(b_{0}, b_{1}\right)\right)<g(\varepsilon)-\gamma, n \geq n_{0} \ldots * *
$$

Using conditions $\left(D_{3}\right)$ and $(*)$,

$$
D\left(b_{n}, b_{n+z}\right)>0, n \geq n_{0} \Rightarrow g\left(D\left(b_{n}, b_{n+z}\right)\right) \leq g\left(\sum_{i=n}^{n+z-1} D\left(b_{i}, b_{i+1}\right)\right)+\gamma<g(\varepsilon)
$$

Thus, we obtain that $D\left(b_{n}, b_{n+z}\right)<\varepsilon, n \geq n_{0}$ by $\left(\mathrm{F}_{1}\right)$. It is seen that this sequence $\left\{b_{n}\right\}$ is an $\mathrm{F}$-Cauchy.

Because of the range of $S$ contains the range of $T$ and the range of at least one is $F$-complete, there exists a $d \in S(X)$ such that $\lim _{n \rightarrow \infty} D\left(S a_{n}, d\right)=0$. Therefore there exists a sequence $\left(x_{n}\right)$ in $[0,+\infty)$ and $x_{n} \rightarrow 0$, $D\left(S a_{n}, d\right) \leq x_{n}$. Moreover, an $e \in X$ can be found, $S e=d$.

Now, show that $T e=d$. Suppose that $D(T e, d)>0$. From the condition $\left(D_{3}\right)$,

$$
g(D(T e, d)) \leq g\left(D\left(T e, T b_{n}\right)\right)+D\left(T b_{n}, d\right)+\gamma, n \in \mathbb{N}
$$

Using condition of theorem and $g$ is non-decreasing,

$$
g(D(T e, d)) \leq g\left[c\left(D(T e, d)+2 D\left(T b_{n}, b_{n}\right)+D\left(b_{n}, d\right)\right)\right]+\gamma, n \in \mathbb{N}
$$

Otherwise, using $\lim _{n \rightarrow \infty} b_{n}=0 \Leftrightarrow \lim _{n \rightarrow \infty} g\left(b_{n}\right)=-\infty$ and $\lim _{n \rightarrow \infty}\left[D(T e, d)+2 D\left(T b_{n}, b_{n}\right)+D\left(b_{n}, d\right)\right]=0$, we obtain that

$$
\lim _{n \rightarrow \infty} g\left(c\left[D(T e, d)+2 D\left(T b_{n}, b_{n}\right)+D\left(b_{n}, d\right)\right]\right)+\gamma=-\infty
$$

This is a contradiction. Consequently, $D(T e, d)=0$, i.e., $T e=d$ and so $d$ is a coincidence point of $T$ and $S$. If $d_{1}$ is another coincidence point, there is $e_{1} \in X$ with $T e_{1}=\mathrm{S} e_{1}=d_{1}$. Therefore,

$$
D\left(d, d_{1}\right)=D\left(T e, \mathrm{~T} e_{1}\right) \leq c D\left(d, d_{1}\right)
$$

Hence, $D\left(d, d_{1}\right)=0$ that is $d=d_{1}$. If $\mathrm{T}$ and $\mathrm{S}$ are weakly compatible, $d$ is a unique common fixed point.

Corollary 3.2. Let $(X, D)$ be an F-metric space. Assume $\mathrm{S}, \mathrm{T}: X \rightarrow X$ provides the below conditions

$i$. For $\forall a, b \in X, D(T(a), T(b)) \leq c D(S(a), S(b))$ where $c<1$,

ii. $T(X) \subset \mathrm{S}(\mathrm{X})$,

iii. $T(X)$ or $\mathrm{S}(\mathrm{X})$ be F-complete subspace.

Therefore, $T$ and $S$ have a unique coincidence point.

Besides, if $T$ and $S$ are weakly compatible, they have a unique common fixed point in $X$.

Example 3.3. Let $X=\mathbb{N}$ be the set of positive real numbers. $D: \mathbb{N} \times \mathbb{N} \rightarrow[0, \infty)$ be the mapping and for all $a, b \in \mathbb{N}$,

$$
D(a, b)=\left\{\begin{array}{cc}
(a-b)^{2}, & a, b \in[0,10] \\
|a-b|, & a, b \notin[0,10]
\end{array}\right.
$$

If take $g(a)=\ln a$, we can show that $D$ be an F-metric by a routine calculation. Next, define $T(a)=a^{2}+1$ and $S(a)=2 a^{2}$. Then, for all $a, b \in \mathbb{N}$ we have

$$
D(T(a), T(b))=\frac{1}{2} D(S(a), S(b)) \leq c D(S(a), S(b)) \text { for } c<1
$$

$T(X)=[1, \infty) \subset[0, \infty)=S(X)$. Moreover, $T(X)$ is an F-complete subspace. That is, all conditions of corollary are satisfied. $T$ and $S$ have a unique coincidence point. 
If $c=2$ be a unique coincident point, $a=1$ and $b=-1$ be coincidence points of $S$ and $T$. But since $T(S(1)) \neq S(T(1)), S$ and $T$ are not weakly compatible, so $S$ and $T$ have no common fixed points.

Theorem 3.4. Let $(X, D)$ be an F-metric, $\mathrm{S}, \mathrm{T}: X \rightarrow X, S^{2}$ be a continuous, and $T$ commute with $\mathrm{S}$. Assume the below conditions is satisfied,

$i$. For all $a, b \in X, D(T(a), T(b)) \leq \alpha u(a, b)$ where $\alpha \in\left(0, \frac{1}{2}\right)$ is a constant and

$$
u(a, b) \in\{D(S(a), S(b)), D(S(a), T(a)), D(S(b), T(b)), D(S(a), T(b)), D(S(b), T(a))\}
$$

ii. $T S(X) \subset S^{2}(X)$,

iii. $T(X)$ or $\mathrm{S}(\mathrm{X})$ be F-complete subspace.

Therefore, $T$ and $S$ have a unique common fixed point.

Proof.

Let $g \in \mathrm{F}, \gamma \in[0, \infty)$ be such that for $\forall a, b \in X$ for every $n \in \mathbb{N}, n \geq 2$ and for every $\left(t_{i}\right)_{i=1}^{n} \subset X,\left(t_{1}, t_{n_{0}}\right)=$ $(a, b)$, we have

$$
D(a, b)>0 \Rightarrow g(D(a, b)) \leq g\left(\sum_{i=1}^{n-1} D\left(\left(t_{i}, t_{i+1}\right)\right)+\gamma\right.
$$

From $F_{2}$, for every sequence $\left\{a_{n}\right\} \subseteq(0,+\infty)$, there exists a $\varepsilon>0$ such that

$$
n \rightarrow \infty \lim _{n \rightarrow \infty} a_{n}=0 \Leftrightarrow \lim _{n \rightarrow \infty} g\left(a_{n}\right)=-\infty \text { and } 0<a<\varepsilon \Rightarrow g(a)<g(\varepsilon)-\gamma
$$

Let $a_{0} \in S(X)$ be arbitrary and $\left\{b_{n}\right\} \subset \mathrm{S}(X)$ be the sequence defined by $S a_{n+1}=T a_{n}=b_{n}$ for $n \in \mathbb{N}$. Now $S b_{n+1}=S T a_{n+1}=T S a_{n+1}=T b_{n}=c_{n}, n \geq 1$. We show that $\left\{c_{n}\right\}$ is an F-Cauchy sequence, so convergent to some c $\in X$. We denote that $S^{2} c=T S c$.

Since $\lim _{n \rightarrow \infty} S b_{n}=\lim _{n \rightarrow \infty} S T a_{n}=\lim _{n \rightarrow \infty} T S a_{n}=\lim _{n \rightarrow \infty} T b_{n}=\lim _{n \rightarrow \infty} c_{n}=c$, it follows that $\lim _{n \rightarrow \infty} S^{4} a_{n}=\lim _{n \rightarrow \infty} S^{3} T a_{n}=$ $\lim _{n \rightarrow \infty} T S^{3} a_{n}=S^{2} c$, since $S^{2}$ is continuous. Thus, we get

$$
D\left(S^{2} c, T S c\right) \leq D\left(S^{2} c, S^{3} T a_{n}\right)+D\left(S^{3} T a_{n}, T S c\right) \leq D\left(S^{2} c, S^{3} T a_{n}\right)+\alpha u_{n}
$$

where $u_{n} \in\left\{D\left(S^{4} a_{n}, S^{2} c\right), D\left(S^{4} a_{n}, T S^{3} a_{n}\right), D\left(S^{2} c, T S c\right), D\left(S^{4} a_{n}, T S c\right), D\left(S^{2} c, T S^{3} a_{n}\right)\right\}$.

Choose any $n_{0} \in \mathbb{N}$, for all $n \geq n_{0}$, since $S^{3} T a_{n} \rightarrow S^{2} c$ and $S^{4} a_{n} \rightarrow S^{2} c$, then we have $D\left(S^{2} c, S^{3} T a_{n}\right) \leq x_{n}$ and $D\left(S^{4} a_{n}, S^{2} c\right) \leq y_{n}$, as $x_{n} \rightarrow 0$ and $y_{n} \rightarrow 0$. We have the five cases:

Case 1: $D\left(S^{2} c, T S c\right) \leq D\left(S^{2} c, S^{3} T a_{n}\right)+\alpha D\left(S^{4} a_{n}, S^{2} c\right) \leq x_{n}+\alpha y_{n}$

Case 2:

$$
\begin{aligned}
D\left(S^{2} c, T S c\right) & \leq D\left(S^{2} c, S^{3} T a_{n}\right)+\alpha D\left(S^{4} a_{n}, T S^{3} c\right) \\
& \leq D\left(S^{2} c, S^{3} T a_{n}\right)+\alpha\left(D\left(S^{4} a_{n}, S^{2} c\right)+\alpha D\left(T S^{3} a_{n}, S^{2} c\right)\right) \\
& \leq x_{n}+\alpha\left(y_{n}+x_{n}\right) \\
& =(1+\alpha) x_{n}+\alpha y_{n}
\end{aligned}
$$

Case 3: $D\left(S^{2} c, T S c\right) \leq D\left(S^{2} c, S^{3} T a_{n}\right)+\alpha D\left(S^{2} c, T S c\right) \leq \frac{x_{n}}{1-\alpha}$ 
Case 4:

$$
\begin{aligned}
D\left(S^{2} c, T S c\right) & \leq D\left(S^{2} c, S^{3} T a_{n}\right)+\alpha D\left(S^{4} a_{n}, T S^{3} c\right) \\
& \leq D\left(S^{2} c, S^{3} T a_{n}\right)+\alpha\left(D\left(S^{4} a_{n}, S^{2} c\right)+D\left(T S c, S^{2} c\right)\right) \\
& \leq \frac{x_{n}+\alpha y_{n}}{(1-\alpha)}
\end{aligned}
$$

Case 5:

$$
\begin{aligned}
D\left(S^{2} c, T S c\right) & \leq D\left(S^{2} c, S^{3} T a_{n}\right)+\alpha D\left(S^{2} c, T S^{3} a_{n}\right) \\
& \leq x_{n}+\alpha x_{n} \\
& \leq(1+\alpha) x_{n}
\end{aligned}
$$

Therefore, $D\left(S^{2} c, T S c\right)=0$ that is $S^{2} c=T S c$. $T S c$ is a common fixed point for $T$ and $S$. Put in the inequality $D(T a, T b) \leq \alpha u(a, b), a=T S c, b=S c$ we get $T(T S c)=T S c$. Since $S^{2} c=T S c$, i.e. $S(S c)=T(S c)$, we have $S(T S c)=T S^{2} c=T(T S c)=T S c$.

\section{Conclusion}

In this work, we present some new common fixed-point theorems in F-metric spaces. Moreover, we give some examples that support our results. Our results extend and generalize the fixed-point theory. We hope that our research results offer a mathematical foundation. In future studies, we will explore the concrete applications of the obtained results.

\section{Author Contributions}

The author read and approved the last version of the manuscript.

\section{Conflicts of Interest}

The author declares no conflict of interest.

\section{References}

[1] S. Czerwik, Contraction Mappings in b-Metric Spaces, Acta Mathematica et Informatica Universitatis Ostraviensis 1(1) (1993) 5-11.

[2] M. A. Khamsi, N. Hussain, KKM Mapping in Metric Type Spaces, Nonlinear Analysis 7(9) (2010) 31233129.

[3] R. Fagin, R. Kumar, D. Sivakumar, Comparing Top k Lists, SIAM Journal on Discrete Mathematics 17(1) (2003) 134-160.

[4] W. Kirk, N. Shahzad, Fixed Point Theory in Distance Spaces, Springer: Cham, 2014.

[5] V. S. Gahler, 2-Metrische Raume und ihre Topologishe Strüktür, Mathematische Nachrichten 26 (1963/1964) 115-118.

[6] Z. Mustafa, B. Sims, A New Approach to Generalized Metric Spaces, Journal of Nonlinear and Convex Analysis 7(2) (2006) 289-297.

[7] S. G. Matthews, Partial Metric Topology. In: Proceedings of the 8th Summer Conference on General Topology and Applications. Annals of the New York Academy of Sciences 728 (1994) 183-197. 
[8] M. Jleli, B. Samet, A Generalized Metric Space and Related Fixed-Point Theorems, Fixed Point Theory and Its Applications Article Number 61 (2015) 1-14.

[9] M. Jleli, B. Samet, On a New Generalization of Metric Spaces, Journal of Fixed-Point Theory and Applications Article Number 12820 (2018) 1-20.

[10] A. L. Alnaser, D. Lateef, A. H. Fouad, J. Ahmad, Relation Theoretic Contraction Results in F-Metric Spaces, Journal of Nonlinear Sciences and Applications 12 (2019) 337-344.

[11] M. Abbas, G. Jungck, Common Fixed-Point Results for Non-Commuting Mappings without Continuity in Cone Metric Spaces, Journal of Mathematical Analysis and Applications 341 (2008) 416-420. 